\title{
Time for analysis
}

\section{Magnesium, oxygen and hydrogen are elements in this week's selection.}

\section{TOC-V analyser}

Shimadzu

www.shimadzu.com

Integrated sample pre-treatment

Shimadzu's TOC-V series laboratory TOC (total organic carbon) analysers incorporate an integrated sample pre-treatment system with an eight-port valve and a syringe with sparging capacity. Acidification and sparging take place within the instrument, eliminating the need for dilution of acid or alkaline samples and reducing the potential for user contact with concentrated acids. With auto-dilution, the instrument is able to measure from $4 \mu \mathrm{g}$ to $25,000 \mathrm{mg}$ per litre. It also performs up to 10-point calibrations using a standard TOC solution. It is available as a stand-alone or as a PC-controlled instrument.

\section{SuperSignal III}

Pierce www.piercenet.com

Speedy, non-radioactive $\mathrm{RPA}$

Pierce has collaborated with Ambion to produce this non-isotopic ribonuclease protection assay (RPA) kit. Exposurescan be madein minutes rather than overnight and probes remain stable for years, rather than weeks, with no need to run probe purifications. The assay uses a single-tube reaction that requires no phenol extraction or proteinase $\mathrm{K}$ digestions. A kit instruction book can be downloaded from Pierce's website.

\section{Micro pH electrode}

Lazar Research Laboratories www.lazarlab.com

\section{Small wonder}

This electrode from Lazar Research Laboratories can measure samples as small as $5 \mu 1$ less than a tenth the volume of a single drop of liquid - as well as small biological specimens down to less than $0.5 \mathrm{~mm}$ in length. The electrode fits directly in 96-well plates, microcentrifuge tubes, serum cups, capillary tubes and NMR tubes. Biological specimens that can be measured indude various animal and plant tissues. Surface $\mathrm{pH}$ measurements on biological and chemical samples can also be made. The probe is made of sturdy fluoropolymer and has a $1 \mathrm{~mm}$ tip with a $2 \mathrm{~mm}$ diameter body. It can be used with any standard $\mathrm{pH}$ meter or stripchart recorder.

\section{Finnpipette Focus}

ThermoLabsystems www.thermo.com Take the strain out of pipetting

The ergonomically designed Focus pipette features a short tip cone, a removable snapon handle and a quick-click volume setting. Its short tip cone improves comfort by minimizinghand and arm strain during pipetting. The handle comes in three sizes, allowing the user to choose which one best fits his or her hand. Volume setting is fast and provides fine

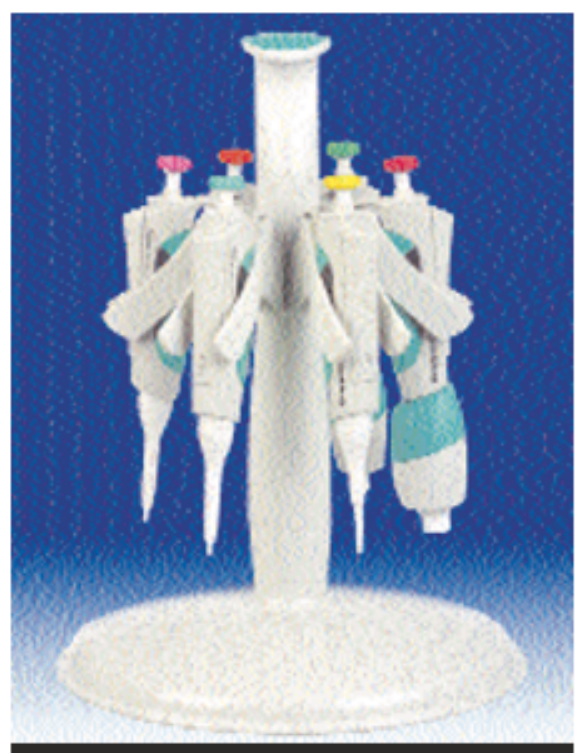

Focus on the rack: all the sizes, in all the colours. control over small increments. The pipette has a soft-touch tip ejector, which ejects the tip with a light push. The pipette is available in 10 volumeranges.

\section{LCpro}

ADCBioscientific www.adc.co.uk Travel light

ADC Bioscientific describes the LCpro as an intelligent, portable photosynthesis measurement system. The unit weighs only $4 \mathrm{~kg}$ and provides automatic and independent control of the environmental conditions within the plant leaf chamber, including $\mathrm{CO}_{2}, \mathrm{H}_{2} \mathrm{O}$, temperature and PAR. It will function for up to 16 hours from a single charge, allowing photosynthesis experiments to be performed in many field sites. A range of interchangeable leaf chambers is available.

\section{SR6189}

\section{Olympus Diagnostic Systems}

www.olympus.com Improved magnesium assay

OSR6189 is a new magnesium reagent developed for use on all Olympus Diagnostics analysers. The reformulated assayuses stable, ready-to-use liquid reagents delivered in barcoded vials for automatic information transfer. The new reagent has a linear range of $0.2-3.3 \mathrm{mmol}$ per litre for serum, increasing the upper limit from the former $2.88 \mathrm{mmol}$ per litre. Decreased blood and tissue levels of magnesium have been associated with high blood pressure, kidney stones, heart disease and heart attacks due to coronary artery spasm. Toxicity, although rare, may cause depression of the central nervous system resulting in symptoms which, in extreme cases, may prove fatal.

These notes are compiled in the Nature office from information provided by the manufacturers. 